\title{
A Morphometric Study of the Maxillary Artery and Lingula in Relation to Mandibular Ramus Osteotomies and TMJ Surgery
}

\author{
Huseyin Avni Balcioglu \\ Cenk Kilic ${ }^{b}$ \\ Altan Varolc \\ Hasan Ozan ${ }^{b}$ \\ Necdet Kocabiyik ${ }^{b}$ \\ Mehmet Yildirim ${ }^{\mathrm{d}}$
}

\section{ABSTRACT}

Objectives: Mandibular osteotomies and resection of the temporomandibular joint (TMJ) ankylosis are the mostly performed surgical procedures in the infratemporal fossa, which is in close proximity with the main trunk of the maxillary artery (MA). It is imperative to avoid the trunk or branches of the maxillary artery, otherwise, massive intraoperative or postoperative hemorrhage may develop. The goal of the study was to investigate the position of the maxillary artery in the infratemporal fossa and the lingula of the mandible.

Methods: Significant landmarks were selected on the mandibles of formalin fixed cadavers, and the distances were measured between the maxillary artery and the bony landmarks with a digital caliper.

Results: The average distances between the MA and the articular eminence, the medial cortex of the mandibular ramus, the inferior border of the pterygoid fovea and the mandibular notch were $1.67 \pm 0.48 \mathrm{~mm}, 5.38 \pm 2.47 \mathrm{~mm}, 16.84 \pm 1.74 \mathrm{~mm}, 2.94 \pm 0.52 \mathrm{~mm}$, respectively. Course pattern of the MA at the subcondylar level was also mapped. In order to determine the position of the lingula, the average distances between the tip of the lingula and the mandibular notch, the inferior border of the ramus, the anterior margin of the ramus and posterior margin of the ramus were measured and found as $15.4 \pm 2.1 \mathrm{~mm}, 49.5 \pm 4.3 \mathrm{~mm}, 18.1 \pm 2.7 \mathrm{~mm}, 16.6 \pm 2.5 \mathrm{~mm}$, respectively. No significant differences were found between the right and left sides, for all parameters.

Conclusions: The studied parameters will assist and navigate clinicians to determine the anatomic proximity to the maxillary artery, and, minimize the risk of damaging the vessel. (Eur J Dent 2010;4:166-169)

Key words: Maxillary artery; Lingula; Mandible; Vertical ramus osteotomy; Sagittal split osteotomy.

- a Department of Anatomy, Faculty of Dentistry, Istanbul University, Istanbul, Turkey.

b Department of Anatomy, Gulhane Military Medical Academy, Ankara, Turkey.

Department of Oral and Maxillofacial Surgery, Faculty of Dentistry, Marmara University, Istanbul, Turkey.

d Department of Anatomy, Cerrahpasa Faculty of Medicine, Istanbul University, Istanbul, Turkey.
- Corresponding author:

Dr. H. A. Balcioglu

Department of Anatomy, Faculty of

Dentistry, Istanbul University,

Capa, 34390, Istanbul, Turkey.

Phone: +902124142020

Fax: +902125312230

E-mail: h.a.balciogluagmail.com 


\section{INTRODUCTION}

Several procedures in maxillofacial surgery are performed in close proximity to the maxillary artery (MA) in the infratemporal fossa. ${ }^{1}$ Considering the fact that MA and its branches are vulnerable to iatrogenic injury during surgical approaches, precautions have to be taken in order to improve intraoperative safety and minimize or prevent massive life-threatening hemorrhage occurring after injury to the MA. ${ }^{2}$ The complicated nature of anatomy makes the first two parts of the MA relatively inaccessible and direct ligation impractical. Surgical exposure of the artery without damaging the facial nerve and condylar process is difficult. $^{3}$

Sagittal split ramus osteotomy (SSRO) or intraoral vertical ramus osteotomy (IVRO), release of TMJ ankylosis, mandibular anesthetic block techniques, and drilling sequence during internal rigid fixation for subcondylar fractures have the potential to damage the MA. ${ }^{4-7}$ The MA emerges from the external carotid artery at the subcondylar level and passes in anteromedial fashion deep to the condylar neck of the mandible into the infratemporal fossa. ${ }^{8}$ Although the proximity of the artery to the medial cortex of the condylar process varies in the reported studies, it is known to pass immediately over the periosteum of the subcondylar area. The course of the MA differs according to race. It was found to pass laterally and superficially to the lateral pterygoid muscle in $9 \%$ to $55 \%$ of whites, in $69 \%$ of blacks, and in about $90 \%$ of Japanese. ${ }^{9}$

The maxillary artery is the major vessel with high arterial flow responsible for massive hemorrhage and formation of false aneurism in maxillofacial surgery, including both mandibular and maxillary surgeries. 1,2,10 The branching pattern in the infratemporal/ pterygopalatine fossa, approaches for ligation, etc., were studied in detail; however, a small amount of data exist concerning its spatial relation to mandibular ramus osteotomies and temporomandibular joint surgeries. ${ }^{3,8,9}$ Therefore, we aimed to designate multiple bony landmarks belonging to the mandibular ramus and the glenoid fossa of the temporal bone and describe their morphometric properties to the maxillary artery.

When performing the SSRO, the exact location of the lingula is crucial, since the osteotomy is performed at the region around the lingula of the mandible during the medial horizontal osteot- omy. ${ }^{11}$ We also morphometrically determined the position of the lingula.

\section{MATERIALS AND METHODS}

The maxillary arteries were dissected bilaterally using a lateral infratemporal approach in 17 formalin-fixed adult cadavers 14 males and $3 \mathrm{fe-}$ males, total 34 sides) under a dissection microscope (Stemi 2000, Carl Zeiss, Jena, Germany). The mean age of the cadavers was 54 (range 23-76 years). The covering soft tissues, superficial lobe of the parotid gland, and masseter muscle were removed to unearth the mandibular condyle. The lateral surface of the ramus of the mandible was exposed along with the external carotid artery and the origin of the maxillary artery. Following the removal of the zygomatic arches using an oscillating saw, a mandibular osteotomy was performed, while making sure that the TMJ and the attachment of the lateral pterygoid muscle to the pterygoid fovea were preserved.

The most inferior point of the articular eminence, the medial cortex of the mandibular ramus, the inferior border of pterygoid fovea, and the mandibular notch were the bony landmarks selected to be studied with the maxillary artery. The topographical relationships between the MAs and these landmarks were evaluated. The distances between the defined landmarks and the maxillary arteries were measured using a digital caliper.

In order to determine the exact localization, the condylar level of the MAs was investigated. An imaginary line passing transversely from the inferior border of the pterygoid fovea at the middle of the neck of the mandible was decided as midcervical level. The regions above and below the line were defined as supracervical and infracervical, respectively (Figure 1).

The location of the mandibular lingula was determined by measuring the distance between the tip of the lingula and the sigmoid notch, the inferior border of the ramus, the anterior margin of the ramus, and the posterior margin of the ramus.

All data were analyzed by using one-way analysis of variance (ANOVA) test, with $P<.05$ accepted as significant. Data are presented as mean values $\pm \mathrm{SD}$.

\section{RESULTS}

The MAs coursed horizontally medial to the ramus of the mandible immediately after emerging from the external carotid artery and then ran 
superficial to the inferior head of the lateral pterygoid muscle in all specimens.

The average vertical distances between the MA and the most inferior point of the articular eminence, the medial cortex of the mandibular ramus, the inferior border of the pterygoid fovea, and the mandibular notch were determined as $1.67 \pm 0.48 \mathrm{~mm}, 5.38 \pm 2.47 \mathrm{~mm}, 16.84 \pm 1.74 \mathrm{~mm}$, $2.94 \pm 0.52 \mathrm{~mm}$, respectively. The course pattern of the MA at the subcondylar level was found to be as follows: $41 \%$ of the MAs passed supracervically ( $n=14), 29.5 \%$ passed midcervically $(n=10)$, and the remaining $29.5 \%$ arteries passed infracervically $(n=10)$. The distances measured between the tip of the lingula and the determined points are summarized in Table 1. No significant difference was computed between the mandibular rami and the glenoid fossa ( $P>$.05).

\section{DISCUSSION}

In our study, we investigated the trajectory of the MA at the infratemporal fossa and the dis-

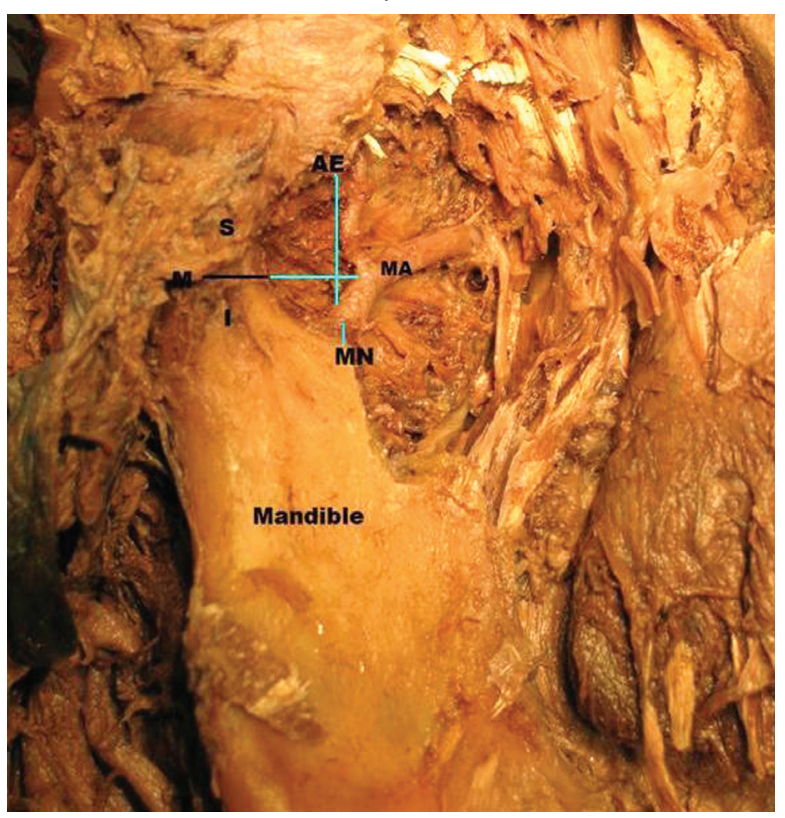

Figure 1. Green lines indicate the measured vertical distances between the MA and selected landmarks. Maxillary artery (MA); Articular eminence (AE); Mandibular notch (MN); Midcervical (M); Supracervical (S); Infracervical (I).

Table 1. The mean distances between the lingula and the specific landmarks for mandibular osteotomy.

\begin{tabular}{lc}
\hline From Lingula to & Distance \\
\hline Mandibular notch & $15.4 \pm 2.1 \mathrm{~mm}$ \\
Inferior border of the ramus & $49.5 \pm 4.3 \mathrm{~mm}$ \\
Anterior margin of the ramus & $18.1 \pm 2.7 \mathrm{~mm}$ \\
Posterior margin of the ramus & $16.6 \pm 2.5 \mathrm{~mm}$ \\
\hline
\end{tabular}

tances between the MA and the bony references on the glenoid fossa and the medial side of the mandibular ramus. To facilitate orientation during ramus osteotomies, the studied parameters were selected as the key bony landmarks of the ramus since these landmarks are used as intraoperative guiding points for bony osteotomies and are dissected to some extent intraoperatively.

The possible risk of injuring the MA is greater in resection of TMJ ankylosis among arthroplastic procedures; since the pattern of ossification and the fibrosis of pericapsular tissues differ greatly in every patient, it is more difficult to predict the course of MA due to anatomical variations and access restrictions. ${ }^{12,13}$ Few cadaver studies have focused on the possible risk of injuring the MA during mandibular ramus osteotomies. ${ }^{9,14}$ The position of the lingula is of critical importance for SSRO, whereas the relevant importance is attributed to the antilingula on the lateral aspect of the ramus for IVRO. ${ }^{15}$

In IVRO, extreme care is required to prevent damage to the maxillary artery when performing bicortical osteotomy from the mandibular notch inferiorly, since the maxillary artery passes upward across the lower head of the lateral pterygoid muscle inside the mandibular notch. 5,9 Therefore, the medial aspect of the mandibular notch should be exposed carefully in the IVRO, and then a Levasseur-Merill or a Bauer retractor should be inserted to protect the MA from any damage. ${ }^{9}$

The reference line for performing the vertical bony cut passes behind the antilingula, which is found approximately with $54 \%$ predominance. The distance from the mandibular notch to the maxillary artery was reported to be $3.3 \pm 1.6 \mathrm{~mm}(\mathrm{n}=8$; range, 2 to $6 \mathrm{~mm}) .^{15}$ These findings were consistent with ours: $2.94 \pm 0.52 \mathrm{~mm}$. We found the ratio of the posterior margin of the ramus to the total ramus width (the sum of the distances between the lingula and the posterior margin of the ramus and anterior margin of the ramus) to be 0.52 . Da Fontoura et $a^{14}$ found a similar value for the ratio of the mandibular foramen-posterior ramus to the total ramus width: 0.32 . The slight difference could be explained by the location of the tip of the lingula, which lies in front of the mandibular foramen.

Behrman ${ }^{16}$ reported severe intraoperative bleeding caused by damage to the MA during an 
SSRO. In a recent review of complications among a group of 655 patients undergoing orthognathic surgery, 78 cases had severe intraoperative bleeding at a volume over 1,000 mL. ${ }^{17}$ Among these, 1 case of an MA tear was the source of severe bleeding during the medial cut of the SSRO. Lanigan ${ }^{2}$ stressed the high probability of severe intraoperative bleeding from injury to the MA when the medial cut of the SSRO was placed in the vicinity of the mandibular notch.

In our cadaveric study, the average distances between the MA and the articular eminence, the articular process, the inferior border of the pterygoid fovea, and the mandibular notch were found to be $1.67 \pm 0.48 \mathrm{~mm}, 5.38 \pm 2.47 \mathrm{~mm}, 16.84 \pm 1.74$ $\mathrm{mm}$, and $2.94 \pm 0.52 \mathrm{~mm}$, respectively. The MA is a potentially high-flow vessel capable of emptying a significant amount of blood within minutes. In the case of MA hemorrhage during TMJ ankylosis, an access restriction generally does not permit direct visualization of the bleeding vessel and requires ligation at the retromolar fossa together with the posterior auricular artery and the superficial temporal artery at the root of the zygoma. $3,8,13$

The measurements in our study revealed that the MA was in very close proximity to the condylar process. The fovea pterygoidea was found to be $16.84 \pm 1.74 \mathrm{~mm}$ away from the MA, which reveals the dangerous proximity of the MA to the upper level of the subcondylar region-the most resected section during ankylosis surgeries. For that reason, subperiosteal dissection of the medial side is of paramount importance to prevent extensive intraoperative or postoperative hemorrhage. The distance from the pterygoid fovea to the MA is approximately equal to the width of a periosteal elevator, which should be placed to keep clearance between the MA and condylar neck in order to prevent any arterial injury.

\section{CONCLUSIONS}

Knowledge of the arterial configuration and key bony structures improves the safety of current treatments and assists in the interpretation of hemorrhagic complications of the maxillary artery. The landmarks we suggest and the distances we measured will assist clinicians in increasing the success of their maxillofacial surgery procedures.

\section{REFERENCES}

1. Lanigan DT, Hey JH, West RA. Major vascular complications of orthognathic surgery: hemorrhage associated with Le Fort I osteotomies. J Oral Maxillofac Surg 1990;48:561573.

2. Lanigan DT. Hey J. West RA. Hemorrhage following mandibular osteotomies. J Oral Maxillofac Surg 1991;49:13-17.

3. Yin NT. Hemorrhage of the initial part of the internal maxillary artery treated by multiple ligations: report of four cases. J Oral Maxillofac Surg 1994;52:1066-1071.

4. Ellis E, Dean J. Rigid fixation of mandibular condyle fractures. Oral Surg Oral Med Oral Pathol 1993;76:6-15.

5. Tuinzing DB, Grebe RB. Complications related to intraoral vertical ramus osteotomy. Int J Oral Surg 1985;14:319-324.

6. Turvey TA, Fonseca RJ. The anatomy of the internal maxillary artery in the pterygopalatine fossa: Its relationship to maxillary surgery. J Oral Surg 1980;38:92-95.

7. Wyatt WM. Sagittal ramus split osteotomy: literature review and suggested modification of technique. $\mathrm{Br} J$ Oral Maxillofac Surg 1997;35:137-141.

8. Rosenberg I, Austin JC, Wright PG, King RE. The effect of experimental ligation of the external carotid artery and its major branches on haemorrhage from the maxillary artery. Int J Oral Surg 1982;11:251-259.

9. Fujimura K, Segami N, Kobayashi S. Anatomical study of the complications of intraoral vertico-sagittal ramus osteotomy. J Oral Maxillofac Surg 2006;64:384-389.

10. Elton VJ, Turnbull IW, Foster ME. An overview of the management of pseudoaneurysm of the maxillary artery: A report of a case following mandibular subcondylar osteotomy. Craniomaxillofac Surg 2007;35:52-56.

11. Kim HJ, Lee HY, Chung IH, Cha IH, Yi CK. Mandibular anatomy related to sagittal split ramus osteotomy in Koreans. Yonsei Med J 1997;38:19-25.

12. Poor D. Pseudoaneurysm of a branch of the maxillary artery following mandibular sagittal split ramus osteotomy: case report and review of the literature. J Oral Maxillofac Surg 2007;65:1807-1816.

13. Peoples JR, Herbosa EG, Dion J. Management of internal maxillary artery hemorrhage from temporomandibular joint surgery via selective embolization. J Oral Maxillofac Surg 1988;46:1005-1007.

14. da Fontoura RA, Vasconcellos HA, Campos AE. Morphologic basis for the intraoral vertical ramus osteotomy: anatomic and radiographic localization of the mandibular foramen. J Oral Maxillofac Surg 2002;60:660-665.

15. Aziz SR, Dorfman BJ, Ziccardi VB, Janal M. Accuracy of using the antilingula as a sole determinant of vertical ramus osteotomy position. J Oral Maxillofac Surg 2007;65:859-862. 
$\square$ A Morphometric Study of the Maxillary Artery and Lingula

16. Behrman SJ. Complications of sagittal osteotomy of the mandibular ramus. J Oral Surg 1972;30:554-561.

17. Panula K, Finne K, Oikarinen K. Incidence of complications and problems related to orthognathic surgery: a review of 655 patients. J Oral Maxillofac Surg 2001;59:1128-1136.

European Journal of Dentistry 